\title{
PERFIL DOS IDOSOS INTERNADOS NO HOSPITAL GERAL EM BELÉM (PARÁ) ${ }^{a}$
}

\author{
Profile of the Elderly Admited in a \\ General Hospital at Belém (Pará - Brazil) \\ Perfil de los Ancianos Internados en el \\ Hospital General en Belém (Pará - Brasil)
}

Maria Izabel Penha de Oliveira Santos

\begin{abstract}
Resumo
0 estudo objetivou identificar o perfil dos idosos internados em um hospital geral da rede pública de saúde em Belém (Pará). Estudo transversal, retrospectivo, analítico. Foram analisadas variáveis sociodemográficas, causas da internação, tempo de permanência, complicações e dependência para cuidados de enfermagem, em 160 prontuários, em 2001. A análise estatística foi processada pelo programa eletrônico Epi-info 6.04. Os resultados apontam: A idade variou entre 65 e 74 anos (43,1\%), e os idosos eram do sexo masculino (53,1\%). A maior freqüência das internações foi por doenças respiratórias (53,7\%); os sintomas evidentes foram dispnéia, hipertemia e hipertensão; e o tempo médio de permanência foi de 16 dias. 0 tempo de internação prolongado aparece como um fator de risco no desenvolvimento de incapacidades, pela imobilidade no leito, complicações como úlceras de decúbito e demais iatrogenias. Houve dependência para os cuidados de enfermagem e necessidade de intervenção interdisciplinar.
\end{abstract}

Palavras-chave: Idosos. Enfermagem. Fatores de Risco.

\begin{abstract}
The study has as objective to identify the profile of the elderly internee in a general hospital of health public service, in Belém (Pará). It was a quantitative, retrospective and descriptive study. It was analyzed social-demographics variables, causes of hospitalization, time of stay, complications and dependence of nursing care in 160 record, 2001. The statistics analysis was made by an electronic program Epi-info 6.04. The results point: The age group was between $64-74$ years old $(43,1 \%)$, and $(53,1 \%)$ was elderly of the male sex, the respiratory diseases were more prevalence $(53,7 \%)$, symptoms evidents were dyspnea, hyperthermia and hypertension, the time stay was 16 days. The hospitalization time look as a factor risk for disability, because the limitation of the bed, wound and other iatrogenics, it was a dependency of nursing care, and necessity of interdisciplinary interview.
\end{abstract}

Keywords:

Elderly. Nursing. Risk factors

\section{Resumen}

El estudio tiene como objetivo identificar el perfil de ancianos hospitalizados en hospital general de salud pública, en Belém, Estado Pará. Estudio cuantitativo, retrospectivo y analitico. Analizadas variables sócio-demográficas, causas de internación, tiempo de permanencia, complicaciónes y dependencias de la enfermería en muestra de 160 historias en 2001. Analisys estadística fué a través del programa electrónico Epi-info 6.04. Los resultados apuntaran: La edad varió de 64 a 74 años $(43$,$) y 85(53,1 \%)$ y los ancianos eran del sexo masculino, la major frecuencia de la internaciónes fueron enfermedades respiratorias $(53,7 \%)$, los síntomas evidentes disnea, hipertermia na hipertensión, tiempo promedio de 16 dias. Los ancianos se internaron en media dos veces al año, tiempo de internación largo aparece como factor de riesgo en el desarrollo de incapacidad, por la inmobilidad en las camas, complicaciónes como úlceras de presion y demás iatrogenias, con dependencia para los cuidados de enfermería y necesidades de intervención interdisciplinar.

Palabras clave:

Ancianos. Enfermería. Factores de Riesgo. 


\section{INTRODUÇÃO}

0 rápido crescimento populacional no Brasil gerou um impacto importante em todos os segmentos da sociedade. Houve mudanças no perfil de morbimortalidade, levando o Brasil a uma chamada "transição", tanto demográfica como epidemiológica, o que aponta para desafios quanto a uma nova forma de "olhar", bem como de "cuidar" da população idosa $a^{1,2,3,5}$.

Devido ao crescente processo de envelhecimento, estimase que, a partir de 2025, o Brasil se torne o sexto país em população na faixa etária de 60 e mais anos de vida. Acreditase que esse fenômeno seja justificado pelas melhorias das condições médico-sanitárias ocorridas ao longo dos anos e que tiveram, como consequêencia, a queda nas taxas de fecundidade e mortalidade ${ }^{1-5}$.

Com efeito, na cidade de Belém, Estado do Pará, a população de idosos vem experimentando um crescimento a partir de 1994, e, em 2001, as pessoas com 60 anos e mais já representavam $6,9 \%$ da população ${ }^{5}$.

Pesquisas vêm mostrando que, qualquer que sejam os indicadores observados na avaliação da qualidade de saúde da população idosa, eles apontam para maior utilização de serviços e custos maiores, quando comparados com os da população mais jovem ${ }^{1,3,4,5}$.

Desse modo, a hospitalização de pessoas idosas passou a ser um motivo de preocupação para os profissionais de saúde, principalmente para os enfermeiros, devido aos possíveis riscos, complicações, tempo de internação e custos que podem surgir dessa modalidade de assistência.

Estudo realizado em hospital geral público, na região Sudeste, apontou para uma alta taxa de internação de pessoas com mais de 60 anos. A maioria dos idosos foi submetida a procedimentos de baixo risco, as doenças cardiovasculares foram as mais prevalentes, e as especialidades cirúrgicas corresponderam a $60,3 \%$ do total das internações. Indicava, porém, que os idosos precisariam ser mais bem avaliados pela equipe de saúde?

Tomando por base essa última afirmação, o objetivo deste estudo foi o de identificar o perfil dos idosos internados em um hospital geral público, na cidade de Belém, Estado do Pará, no intuito de conhecer as causas de internação, as possíveis complicações que surgiram em decorrência da hospitalização, o número de dias de internação e os cuidados de enfermagem dispensados a esses idosos, a partir de sua dependência, assim como os riscos a que estiveram expostos nesse período.

A justificativa para realizar este estudo, na época, foi a de que ainda eram escassas, em nossa Região, pesquisas que informassem sobre essa realidade. Um outro ponto que também considerei foi o de que, até momento da realização deste estudo, não dispúnhamos de um centro de referência de atenção à pessoa idosa, de onde informaçõoes acerca da saúde dessa população pudessem ser obtidas.

Assim, acredito que esta pesquisa venha ao encontro da necessidade de produzir investigações que possam ajudar os enfermeiros e outros profissionais da área da saúde que atuam com pessoas idosas, informando-os sobre as doenças que mais acometem os idosos e que possam levá-los à hospitalização, bem como sobre suas necessidades assistenciais, durante 0 período de internação.

\section{MÉTODO}

0 estudo caracterizou-se como do tipo transversal, retrospectivo, analítico, documental, realizado em prontuários de idosos que estiveram internados no ano de 2001. Foi realizado em um hospital universitário, localizado no bairro do Guamá, na cidade de Belém, Estado do Pará, e que está ligado ao Sistema Único de Saúde (SUS) ${ }^{6,20}$.

0 hospital oferece para internação as especialidades de clínica médica, pneumologia, doenças infecciosas e parasitárias, pediatria, cirurgia, neurologia e doença de Alzheimer. Em nível ambulatorial, fisioterapia respiratória e assistência ambulatorial aos egressos da hospitalização por doenças crônicas. É também um hospital de referência no diagnóstico da Síndrome da Imunodeficiência Adquirida (AIDS) e de doenças respiratórias agudas e crônicas.

A pesquisa foi desenvolvida após aprovação pelo departamento de ensino e pesquisa do hospital, conforme declaração em anexo. Neste ano de 2001 foram hospitalizadas aproximadamente 5.000 pessoas, de todas as faixas etárias, pelo menos 1.000 , dentre elas, na faixa etária de 60 anos ou mais.

Foram considerados, para atender aos critérios de inclusão na pesquisa, os prontuários de pacientes que tivessem idade igual ou superior a 60 anos, de ambos os sexos, hospitalizados por um período superior a 48 horas, entre janeiro e dezembro de 2001. Os que não obedeceram a esses critérios foram excluídos.

Os cálculos foram realizados através da fórmula de distribuição normal, com base no número de internações de idosos, que resultou em um valor aproximado a $300(\mathrm{~N}=300)$. Ou seja, a amostra constou de 300 prontuários de pacientes internados no período (janeiro a dezembro de 2001), e, destes 300 prontuários, somente 160 atendiam aos critérios de inclusão da pesquisa, tendo sido excluídos os demais.

Foi solicitada, inicialmente, ao Departamento de Arquivo Médico e Estatístico (DAME) da instituição, uma listagem com o número de matrícula dos pacientes, a clínica em que haviam sido admitidos para tratamento e as datas da admissão e alta no hospital.

Para a obtenção dos dados, foi construído um instrumento subdividido em três partes. Na primeira, foi dada ênfase às variáveis sociodemográficas, como sexo, idade, procedência, estado civil e profissão. Na segunda constavam as informações sobre a hospitalização, como diagnóstico médico, tempo de internação, número de internações no ano, procedimentos e os tipos de cuidados registrados pela equipe de enfermagem. E uma terceira parte foi reservada para observações necessárias, que não tivessem sido contempladas nas partes anteriores, como a classificação da doença pelo CID-10, tipo de alta e custo da internação, conforme os registros na Autorização de Internação Hospitalar - Sistema Único de Saúde (AIH-SUS)?

Os dados foram processados no programa eletrônico Epiinfo 6.04, e as tabelas e gráficos, construídos no Microsoft Excel 2000, com a descrição apenas das freqüências simples, expressas em percentuais. 


\section{RESULTADOS E DISCUSSÃO}

A Tabela 1 evidenciou que, entre os idosos da amostra, e que foram internados no período da pesquisa, 53,1\% eram do sexo masculino. A faixa etária de maior freqüência ficou entre 65 e 74 anos de idade $(43,1 \%)$.

Quanto à questão do gênero, os indicadores demográficos populacionais, no Brasil, apontam que os idosos do sexo masculino apresentam maiores riscos de adoecer e morrer em relação aos do sexo feminino, com as mulheres apresentando uma expectativa de vida de 10 anos a mais que os homens ${ }^{3,8}$.

Estudo sobre a freqüência de internação de homens, em relação a mulheres, informa que ela é progressivamente maior nos homens dos 20 aos 69 anos de idade. E se torna menor, a partir dos 80 anos, pois o predomínio, a partir dessa idade, passa a ser das internações de mulheres ${ }^{5}$.

Sobre a questão etária, ficou caracterizado que, neste estudo, os idosos ainda eram jovens, segundo a classificação proposta pela Organização das Nações Unidas (ONU), que divide os idosos em três categorias: os pré-idosos (entre $55 \mathrm{e}$ 64 anos); os idosos jovens (entre 65 e 79 anos) e os idosos de idade avançada (com mais de 75 ou 80 anos). Estes últimos, com mais de 80 anos, são e vão continuar sendo do sexo feminino (IBGE) $)^{1,4}$.

Tabela 1:

Distribuição da freqüencia por sexo, faixa etária e estado civil dos idosos internados em hospital geral. Belém/Pará - 2001.

\begin{tabular}{l|c|c}
\hline IDENTIFICAÇÃO & $\mathbf{N}=160$ & $\mathbf{\%}$ \\
\hline SEXO & & \\
Masculino & 85 & 53,1 \\
Feminino & 75 & 47,8 \\
IDADE (em anos) & & \\
$<65$ & 47 & 29,3 \\
65 a 74 & 69 & 43,1 \\
75 a 84 & 31 & 19,3 \\
$>=85$ & 13 & 8,1 \\
ESTADO CIVIL & & \\
Casado & 68 & 42,5 \\
Viúvo & 39 & 24,4 \\
Solteiro & 34 & 21,3 \\
\hline
\end{tabular}

Em relação à clínica onde os idosos foram admitidos para tratamento, neste estudo, a maior freqüência das internações dos idosos foi na clínica de doenças respiratórias - pneumologia $(53,7 \%)$, muito embora $28,1 \%$ dos pacientes tenham sido submetidos a algum tipo de tratamento cirúrgico, como evidenciado na Tabela 2.

A especificidade do hospital em doenças do aparelho respiratório e o fato de ser ele um hospital de referência para essas patologias na região Norte foram decisivos para essa freqüência de internações na referida clínica, assim como a
Tabela 2:

Distribuição da freqüencia por clínica e segundo o sexo dos idosos internados em hospital geral. Belém/Pará - 200.

\begin{tabular}{l|cc|cc|c|c}
\hline \multirow{2}{*}{ CLÍNICA } & \multicolumn{4}{c|}{ SEXO } & \multicolumn{2}{c}{ TOTAL } \\
\cline { 2 - 6 } & \multicolumn{3}{|c|}{ Masculino } & \multicolumn{2}{c|}{ Feminino } & \multicolumn{2}{c}{} \\
\cline { 2 - 6 } & Freq. & $\%$ & Freq. & $\mathbf{\%}$ & Freq. & \% \\
\hline Pneumologia & 44 & 51,7 & 42 & 56,0 & 86 & 53,7 \\
Cirúrgica & 24 & 28,2 & 21 & 28,0 & 45 & 28,1 \\
Médica & 9 & 10,5 & 9 & 12,0 & 18 & 11,2 \\
DIP & 8 & 9,4 & 3 & 4,0 & 11 & 6,8 \\
TOTAL & $\mathbf{8 5}$ & $\mathbf{5 3 , 1}$ & $\mathbf{7 5}$ & $\mathbf{4 6 , 8}$ & $\mathbf{1 6 0}$ & $\mathbf{4 6 , 2}$ \\
\hline
\end{tabular}

prevalência das doenças respiratórias para essa faixa etária. Um outro fator, que contribuiu também para a demanda na clínica de pneumologia, está relacionado às condições climáticas da região - extremamente quente e úmida - o que influencia a agudização das doenças crônicas respiratórias ${ }^{9}$.

Com efeito, as doenças pulmonares obstrutivas crônicas (DPOC) aparecem como a segunda causa de morte em idosos, após as doenças cardiovasculares, e, de certa forma, essa duas patologias podem se manifestar associadas nos idosos ${ }^{9-10}$.

A Tabela 3 faz referência à distribuição das freqüências dos procedimentos realizados nos idosos durante o período de sua internação. Nesse aspecto, para os que apresentavam quadro clínico compatível com doenças respiratórias crônicas, foi possível observar que, nos procedimentos em que houve a participação da enfermagem, destacaram-se a oxigenoterapia e nebulização, indicados para esses casos. Eles foram prestados a 78,1\% e $62,5 \%$ dos pacientes, respectivamente.

\section{Tabela 3:}

Distribuição da freqüencia dos procedimentos a que foram submetidos os idosos durante a internação em Hospital Geral Belém/Pará - 2001.

\begin{tabular}{l|c|c}
\hline PROCEDIMENTOS & Freqüência & (N $=160)$ \\
\hline Higiene Oral e Corporal & 145 & 90,6 \\
Oxigênio & 125 & 78,1 \\
Nebulização & 100 & 62,5 \\
Curativos & 72 & 45,0 \\
Sondagens & 13 & 8,1 \\
Biópsias & 9 & 5,6 \\
Endoscopia & 7 & 4,4 \\
Gasometria & 6 & 3,7 \\
Broncoscopia & 5 & 3,1 \\
Toracocentese & 2 & 1,2 \\
\hline
\end{tabular}

Convém ilustrar aqui o papel da enfermagem, na avaliação clínica do paciente portador de doença crônica e na tomada de decisões, implementando adequadamente a assistência, no sentido de promover o bem-estar dos pacientes, através de procedimentos que ajudem na manutenção do fluxo aéreo adequado. 
Com relação aos sinais e sintomas apresentados pelos idosos e sua freqüência, durante esse período de internação, eles se encontram destacados na Tabela 4.

\section{Tabela 4:}

Distribuição da freqüencia dos sinais e sintomas em idosos internados em Hospital Geral - Belém/Pará - 2001.

\begin{tabular}{l|c|c}
\hline SINAIS E SINTOMAS & Freqüência & ( $\mathbf{\%}$ \\
& & $\mathbf{1 6 0})$ \\
\hline Dispnéia & 106 & 66,2 \\
Hipertemia & 97 & 60,6 \\
Tosse Progressiva & 91 & 56,8 \\
Hipertensão & 78 & 48,7 \\
Edema MMII & 45 & 28,1 \\
Escarro de Sangue & 15 & 9,3 \\
Icterícia & 12 & 7,5 \\
\hline
\end{tabular}

A dispnéia foi um sintoma freqüente em $66,2 \%$ dos idosos, já que um número considerável desses pacientes foi internado na clínica de pneumologia. Esse sintoma, em geral, está presente e aparece em função da descompensação nos pacientes obstrutivos crônicos e também nos quadros clínicos de Insuficiência Cardíaca Esquerda, podendo ser noturno, no idoso, o que sugere um sono intranqüilo, com pesadelos. Está associado a outras condições clínicas, como em pós-operatório, no tromboembolismo pulmonar, ou também pode surgir de forma súbita, o que é caracterizado, em idosos, como uma manifestação solitária de angina ou infarto do miocárdio, e ainda por ser manifestação de edema agudo de pulmão ${ }^{10-11}$.

Dessa forma, sintomas agudos ou crônicos, como a dispnéia, em pacientes idosos, precisam ser alvo de avaliação constante pela equipe de enfermagem, devido às complicações que podem estar associadas.

Um outro sintoma presente nos idosos da amostra, durante a internação, foi a hipertermia em $60,6 \%$ dos pacientes. Esse sintoma, em idosos, geralmente está associado a processos infecciosos ou inflamatórios, sendo então motivo de preocupação, pois, no curso desses processos, pode estar mascarado um quadro clínico de delirium que, muitas vezes, passa despercebido ou pode ser confundido com alterações do processo do envelhecimento ${ }^{11-17}$.

A hipertensão arterial (HA) também foi um dado nosológico importante, que esteve presente em $48,7 \%$ da amostra. Estudos informam que a HA é um fator de risco para a saúde do idoso, podendo vir a desenvolver eventos cardiovasculares, como: acidente vascular cerebral, infarto do miocárdio, insuficiência cardíaca, insuficiência renal, aneurisma de aorta, insuficiência vascular periférica, independente de sexo ou raça, em termos de risco relativo, risco absoluto, gradiente de risco e risco atribuível ${ }^{10-6}$.

Com efeito, segundo estudo realizado na população do Rio de Janeiro, as doenças que mais acometem os idosos são as de características crônicas, que podem perdurar por 15, 20 ou mais anos, e que deverão consumir mais recursos da área da saúde. Porém, nem sempre esses custos são revertidos adequadamente às suas necessidades, e essa inadequação não ocorre somente no serviço público ${ }^{12}$.

0 curso das doenças crônicas que acometem os idosos é um dos fatores que favorecem um tempo de internação mais longo para esse grupo. Ou seja, as pessoas acima de 60 anos levam um tempo maior para produzir as respostas do seu sistema imunológico, bem como maior tempo para as respostas terapêuticas, em relação aos mais jovens. Isto se deve ao processo normal de envelhecimento, que tende a ficar mais comprometido quando essas doenças estão presentes.

Nesta pesquisa, foi encontrado um tempo médio de 16 dias de internação, e $25 \%$ dos idosos tiveram um tempo de internação entre 11 e 20 dias. Sobre esse aspecto, foi possível pensar nos riscos e complicações advindas de um período longo de internação, provocados pela imobilidade no leito, como é caso da formação de úlceras de decúbito, piora das condições respiratórias, exposição maior às infecções hospitalares e ansiedade pelo afastamento familiar, que podem agravar ainda mais o quadro clínico dos idosos (Tabela 5).

Pelos registros obtidos nos prontuários individuais, empiricamente foi possível inferir que o retardo nos procedimentos complementares de diagnóstico foi também um outro fator que contribuiu para um tempo prolongado de internação, além de gerar elevação dos custos com as diárias hospitalares.

\section{Tabela 5:}

Distribuição da freqüencia dos idosos internados quanto ao tempo de permanência em Hospital - Belém/Pará - 2001.

\begin{tabular}{c|c|c}
\hline $\begin{array}{c}\text { TEMPO DE } \\
\text { PERMANÊNCIA } \\
\text { (em dias) }\end{array}$ & Freqüência & $\%$ \\
\hline 1 a 10 & 92 & 57,5 \\
11 a 20 & 40 & 25,0 \\
21 a 30 & 11 & 6,9 \\
31 a 40 & 12 & 7,5 \\
> 40 & 5 & 3,1 \\
\hline TOTAL & 160 & 100,0 \\
\hline MÉDIA GERAL & 16,0 & - \\
\hline
\end{tabular}

Um outro fator considerado nas pesquisas sobre hospitalização de idosos é a iatrogenia, que pode ser causada pela própria equipe multiprofissional, decorrente das diferentes formas de assistência prestadas aos pacientes. Esse conceito, em geriatria, pode ter um significado amplo, quando relacionado às condutas tomadas por vários membros da equipe ${ }^{13-14}$.

Sobre esse aspecto, alguns autores citam como complicações as iatrogenias de qualquer natureza, sendo importantes na pessoa idosa, dentre elas, a própria dificuldade de adaptação ao meio, maior vulnerabilidade do idoso às agressões, incluindo intervenções propedêuticas e terapêuticas, e incidência maior de doenças crônicas, tornando-se necessário uso maior de drogas. Também são incluídas a plurimorbidade, procedimentos invasivos - 
diagnósticos e terapêuticos, uso incorreto de medicações e déficits sensoriais e mnemônicos ${ }^{13,14,16-17}$.

Quanto aos procedimentos realizados durante a internação dos idosos, ficou mais evidente nos registros dos prontuários analisados a assistência realizada pela equipe médica e de enfermagem, em relação aos demais profissionais da área de saúde.

Sobre esse aspecto, é proposta do Plano Nacional de Atenção à Pessoa Idosa (PNAPI), que as pessoas idosas sejam assistidas por uma equipe multiprofissional, pois elas estão, cada vez mais, preenchendo os leitos hospitalares e estão carentes dessa forma de assistência ${ }^{19}$.

É proposta também, dessa política, que os serviços de saúde elaborem protocolos de assistência voltados para as pessoas idosas, levando em conta as características dessa faixa etária. Nos prontuários analisados neste estudo, não foi observado nenhum item específico voltado ao paciente geriátrico. Muito embora a análise de tais protocolos não tenha sido um dos objetivos do estudo, identifiquei essa lacuna.

0 quadro clínico apresentado pelos idosos, em decorrência das doenças agudas ou crônicas durante a internação, compromete, muitas vezes, suas condições físicas e cognitivas para o autocuidado. A enfermagem, nesse momento, assume papel importante quanto à ajuda na manutenção da autonomia e independência dos idosos. As alterações para o autocuidado que foram apresentadas pelos componentes deste estudo, durante a internação, encontram-se distribuídas na Tabela 6.

\section{Tabela 6:}

Distribuição da freqüencia das alterações para o autocuidado apresentado pelos idosos internados em Hospital Geral Belém/Pará - 2001

\begin{tabular}{c|c|c}
\hline $\begin{array}{c}\text { ALTERAÇÕES PARA } \\
\text { AUTOCUIDAD0 }\end{array}$ & Freqüência & \% \\
\hline (N = 147) \\
\hline Alimentação & 124 & 84,3 \\
Deambulação & 117 & 79,5 \\
Higiene & 104 & 70,7 \\
Vestir-se & 4 & 2,7 \\
\hline
\end{tabular}

Pelas descrições da equipe de enfermagem, as necessidades mais freqüentes dos idosos foram quanto à alimentação $(84,3 \%)$, deambulação $(79,5 \%)$ e realização de higiene pessoal $(70,2 \%)$, exigindo cuidados específicos de enfermagem, mesmo que temporariamente.

Nesse patamar, destaco a participação da enfermagem na atenção para com as pessoas que envelhecem, principalmente as fragilizadas e que necessitam desses cuidados durante a hospitalização, ajudando na manutenção da independência e qualidade de vida, em especial dos portadores de doenças crônicas.

Estudos vêm sendo realizados, no sentido de enfatizar os cuidados de enfermagem ao paciente idoso, com fundamentos no campo da gerontologia e na habilidade de aplicação de técnicas de tratamentos geriátricos específicos. Como exemplo, cito casos de imobilidade, incontinência urinária, instabilidade postural e riscos de quedas ${ }^{20}$.
Nesse contexto, pesquisas referem que, entre os mais importantes indicadores de saúde na velhice, estão as capacidades funcional e cognitiva. E que a capacidade funcional está relacionada com as condições do indivíduo para se adaptar aos problemas cotidianos. Ou seja, trata-se daquelas capacidades que lhe são requeridas pelo ambiente em que vive ${ }^{21-22}$.

As capacidades funcional e cognitiva, em idosos, estão diretamente relacionadas à autonomia e independência. Trata-se da capacidade de autogoverno, de liberdade e de administrar sua própria vida. Porém, quando alteradas, seja por doença ou por outra condição, isto os limita e, às vezes, até os exclui do meio social.

Uma outra dimensão que se pode acrescentar à perda da capacidade funcional é a fragilidade, entendida como uma vulnerabilidade que o indivíduo apresenta aos desafios do próprio ambiente. Nestes, estão incluídas as condições materiais, econômicas e sociais da pessoa, que possam levá-la à dependência. As repercussões da hospitalização são seguidas, em geral, por uma diminuição da capacidade funcional e por mudanças na qualidade de vida, muitas vezes irreversíveis ${ }^{14,21-22}$.

Em relação ao tipo de alta médica evidenciada, a "melhorada" esteve presente na maioria dos idosos da amostra. 0 custo médio das internações, no período, foi de $\mathrm{R} \$ 406,13$, porém não foi possível, naquele momento, estabelecer comparações com os custos para outras faixas etárias, pois as demais faixas etárias não foram objeto deste estudo, além de existirem limitações quanto a registros que tornassem possível realizar um análise mais detalhada sobre esses custos. No entanto, estudos demonstram que a faixa etária de 60 anos e mais é a que consome os maiores recursos do SUS 1,3,8,5, 16,18,21.

\section{CONCLUSÕES}

0 presente estudo foi realizado com o propósito de identificar o perfil dos idosos internados em 2001 em um hospital de referência na cidade de Belém. Dos 160 prontuários de idosos que foram internados nesse período, $53,1 \%$ eram do sexo masculino, e, entre os idosos da amostra, $43,1 \%$ estavam na faixa etária de 65 a 74 anos de idade.

Os resultados do estudo demonstraram que os sintomas apresentados pelos idosos, durante a internação, eram compatíveis com o quadro clínico de doenças respiratórias crônicas, como a dispnéia $(66,2 \%)$, hipertermia $(60,6 \%)$, tosse progressiva $(56,8 \%)$ e hipertensão arterial $(48,7 \%)$.

Outro fato considerado importante no estudo foi o tempo longo de internação dos idosos, em média 16 dias, que não difere dos perfis nacionais. Esse período prolongado de internação concorre para o estabelecimento de incapacidades, maior exposição a risco de infecção hospitalar, entre outras complicações que podem refletir na recuperação das pessoas idosas.

Os idosos deste estudo apresentaram incapacidades para realização de atividades básicas da vida diária, como alimentação $(84,3 \%)$, deambulação $(79,5 \%)$ e higiene $(70,7 \%)$. Isto implicou em uma avaliação adequada pela equipe de enfermagem, que teve participação importante, realizando intervenções e os cuidados necessários para suprir tais 
incapacidades, muito embora elas possam ter sido geradas pela agudização das próprias doenças. Quanto a esse aspecto, é preciso entender que a identificação e reabilitação precoce de problemas, nessa população, se tornam imprescindíveis para a prevenção de novas incapacidades.

Um dos fatores limitantes para o estudo foi quanto aos registros deficientes e incompletos dos profissionais nos prontuários individuais dos pacientes, prejudicando a coleta de dados, impedindo que a análise de uma amostra maior pudesse ter sido realizada. A grafia pouco legível foi outro aspecto que, muitas vezes, tornou incompreensível 0 entendimento das informações.

\section{Referências}

1. Instituto Brasileiro de Geografia e Estatística -IBGE. Perfil dos idosos responsáveis por domicílio no Brasil 2000. Rio de Janeiro (RJ); 2000.

2. Motta LB. Levantamento do perfil dos idosos internados em hospital geral: análise do processo de internação frente às demandas da população geriátrica. Textos sobre envelhecimento 2001 ago/dez; 3(6): 47-77.

3. Camarano AA. Envelhecimento da população brasileira: uma contribuição demográfica. In: Freitas EV et al,organizadores. Tratado de Geriatria e Gerontologia. Rio de Janeiro(RJ):Guanabara Koogan; 2002.

4. Mendes MRSSB, Gusmão JL, Faro AGM , Leite RCBO. A situação do idoso no Brasil: uma breve consideração. Acta Paul Enferm 2005 18(4): $422-26$

5. Chaimowicz F. Os idosos brasileiros no século XXI: demografia, saúde e sociedade. Belo Horizonte (MG):Posgraduate; 1999.

6. Rea LM, Parker Metodologia da pesquisa:do planejamento à execução. Tradução de Nivaldo Montiguelli Junior. São Paulo (SP): Pioneira; 2000.

7. Organização Mundial da Saúde-OMS. CID-10: Classificação estatística internacional de doenças e problemas relacionados à saúde. Tradução do Centro Colaborador da OMS para a classificação de doenças em português. $3^{\text {a }}$ ed. São Paulo (SP): USP;1996

8. Chaimowicz. F. Epidemiologia e o envelhecimento no Brasil. In: Freitas EV et al, organizadores. Tratado de Geriatria e Gerontologia. Rio de Janeiro(RJ): Guanabara Koogan; 2006.

9. Costa MFL, Guerra HL, Barreto SM, Guimarães RM.Diagnóstico da situação de saúde da população idosa brasileira: um estudo da situação da mortalidade e das internações hospitalares públicas. Inf Epidemiol SUS out/dez: 2000; 9(1), 23-41.

10. Lourenço RA, Veras RP, Silva NAS. Hipertensão arterial e outros fatores de risco de doença cardiovascular: prevalência em uma população de idosos.In: Veras RP.Terceira idade: gestão contemporânea em saúde. Rio de Janeiro(RJ): Relume Dumará; 2002
Devido ao crescimento populacional na faixa etária de 60 anos e mais, maior contingente de pessoas nessa faixa irá, forçosamente, procurar assistência hospitalar. Sobre esse aspecto, sugiro a adequação pelos serviços, nos prontuários de itens e/ou protocolos que identifiquem características físicas e cognitivas do paciente geriátrico.

Acredito que essa discussão não finda aqui e que os resultados deste estudo possam incentivar novas pesquisas pelos enfermeiros na área geronto-geriátrica e demais profissionais de saúde. Principalmente sobre pessoas que envelhecem na Região Norte, carentes ainda de uma sistematização para sua assistência.

11. Costa MFL, Guerra HL, Firmo JOA, Uchôa E.Projeto Bambuí: um estudo epidemiológico de características sócio-demográficas, suporte social e indicadores de condição de vida de saúde em idosos em comparação com adultos jovens. Inf Epidemiol SUS: 2001 out/dez; 10(4): 147-61.

12. Ribeiro EE, Guimarães RM, Toledo AV. Sinais e sintomas do aparelho respiratório. In: Guimarães RM, Cunha UGV. Sinais e sintomas em geriatria. São Paulo(SP): Atheneu; 2004.

13. Veras R P. Terceira idade: gestão contemporânea em saúde. Rio de Janeiro(RJ): Relume-Dumará; 2002.

14. Kane RL, Ouslander JG, Abrass IB.0 essencial em clínica geriátrica Tradução de Martha Müller Romiti Araf. São Paulo(SP): Andrei;1985.

15. Giacomin KC. A iatrogenia na atenção médica aos idosos [atualização científica]. Med CF; 2002 out ; 17(138):13-4

16. Veras RP. Em busca de uma assistência adequada à saúde do idoso: revisão de literatura e aplicação de um instrumento de detecção precoce e previsibilidade de agravos. Cad Saúde Pública 2002 jun; 19(3): 705-15

17.Elie M, Cole MG, Primeau FJ, Bellavance F Delirium risk factors in elderly hospitalized patients. J Gen Intern Med 1998 May; 13: 204-12.

18.Siqueira AB,Cordeiro RC, Perracini MR, Ramos LR.Impacto funcional da internação hospitalar de pacientes idosos. Rev Saúde Pública 2004 out; 38(5): 687-94

19.Lei 8.842, de 4 de janeiro de 1994.Dispõe sobre a política nacional de atenção ao idoso, cria o Conselho Nacional do Idoso e da outras providências. [on-line] Brasília,1994. [citado 5 nov 2002].Disponível em: http//www.planalto.gov.br/ccivil-3/leis/

20.Santos I, Clos AC. Pesquisa quantitativa e metodologia. In: Santos I et al. Pesquisa em enfermagem: novas metodologias aplicadas. Rio de Janeiro (RJ): Guanabara Koogan:1998

21.Caldas CP. Conversando sobre o autocuidado: a qualidade de vida como meta. In: Pacheco JL, Sá JLM, Py L, Goldman SN, organizadores. Tempo rio que arrebata. São Paulo (SP):Setembro; 2005 
22.Veras RP. 0 custo hospitalar crescente no setor público: conseqüência do envelhecimento populacional. In: Terceira idade gestão contemporânea e saúde. Rio de Janeiro (RJ): Relume Dumará; 2002

\section{Nota}

aEste artigo é fragmento da monografia do Curso de Especialização Envelhecimento e Saúde do Idoso, realizado na Faculdade de Ciências Médicas de Belo Horizonte-MG

\section{Sobre a Autora}

\section{Maria Izabel Penha de Oliveira Santos}

Enfermeira, Ms. em Educação, Gerontóloga pela SBGG. Coordenadora do Projeto de Extensão Universidade Aberta à Terceira Idade. Membro do Grupo de Pesquisa-Saúde do Adulto e Idoso da Universidade do Estado do Pará (UEPA). Professora do curso de EnfermagemUniversidade do Estado do Pará. e-mail:princesa@nautilus.com.br 